\title{
Pedunculated gliomas in the lateral ventricle
}

\author{
A. KUNICKI \\ From the Clinic of Neurological Surgery, Cracow Academy of Medicine, and the Laboratory of Neurosurgical \\ Pathology, the Polish Academy of Science, Cracow
}

The name of 'subependymoma' was given by Scheinker in 1948 to a particular variety of glioma found in the vicinity of the fourth ventricle and of the lateral ventricles. These tumours are distinguished by their dense, hard consistency. They are sharply demarcated from their surroundings, and as a rule develop into the lumen of the ventricle. They are intimately connected with the ependyma, seldom grow beyond the fundus of the ventricle, and remain within the ventricle. On the surface and in section, they are of a greyish-brown colour. The tissue is composed of clustered chains of small nuclei, which in transverse section give a picture of nests of cells. No mitoses or polynuclear cells are found. Scheinker's designation of this tumour reflects his views on its cytogenesis. In spite of its distinct morphological peculiarity, Russell and Rubinstein (1959) consider it to be a variety of ependymoma, in which the growth of the ependymal fibrillary glia has dominated the histological picture. Zülch (1956) also sees no justification for differentiating these tumours as a separate variety, and considers them to be ependymomas which have undergone a 'specific retrograde metamorphosis'.

French and Bucy (1948) reviewed their cases of glioma of the septum pellucidum, and in three cases found tumours of such a type, but preferred to use the term 'subependymal astrocytoma'. Chason (1956) described 19 cases, 17 of which had developed from the walls of the fourth ventricle; in many cases there were multiple tumours, even up to the number of eight in one extreme case. In one case, a small tumour protruded into the polar part of the lumen of a lateral ventricle, and in another the tumour emerged from the septum pellucidum, as in the three cases reported by French and Bucy.

Boykin, Cowen, Iannucci, and Wolf (1954) described nine cases belonging to the same morphological group as 'subependymal glomerate astrocytoma', in this way stressing the accumulations of cells in glomeruli or columns separated from one another by the non-cellular fibrillary fields characteristic of these tumours. Six tumours were located in the fourth ventricle, one in a lateral ventricle, and two were intramedullary. In three cases (as in
Chason's), the tumour was unexpectedly discovered at necropsy, and in one case there were two tumours. In Chason's material the tumours were also multiple in four cases. Boykin estimated the total number of tumours described up to 1954 as 21 , which certainly does not indicate the actual prevalence, but still gives evidence that this group of gliomas is small.

The two cases described here are typical examples of these tumours, and merit attention on account of their anatomical location. In both cases the tumour lay in the anterior part of a lateral ventricle and was joined to the ventricular ependyma by a delicate pedicle as thick as a match stick and about $1 \mathrm{~cm}$. in length. These tumours, identically situated, gave a similar pneumoencephalogram, and as intraventricular, pedunculated gliomas justify the title of this report.

\section{CASE REPORTS}

CASE 1 J.R., aged 49, metal-worker (case no. 370/60), was admitted to the clinic on 13 May 1960 . He had suffered from episodic headache for three years. In the month before admission, the first attack of general convulsions with loss of consciousness occurred. Similar attacks were twice repeated without leaving any neurological deficit.

A preliminary examination of the internal organs showed no deviations from the normal. Neurological examination showed a very slight paresis of the labial branch of the right facial nerve and bilateral papilloedema with haemorrhages. Beyond this, no disturbances were found in movement, sensation, or reflexes. Equilibrium, both at rest and during movement, was well preserved, the patient was orientated as to time and place and his behaviour was appropriate to the occasion. Arteriography did not show any changes in the location of the venous or arterial vessels. Air encephalography was therefore carried out, and the result was quite unexpected (Fig. 1). Both lateral ventricles were markedly enlarged, and the third was not demonstrated. In addition, the lower part of the right lateral ventricle showed a filling defect in the lumen which, together with the absence of air in the third ventricle, indicated the presence of a tumour occupying the anterior part of the third ventricle and protruding into the lateral ventricle. On 24 May 1960 the cranium was opened by a flap revealing the right fronto-temporal region. The lateral 


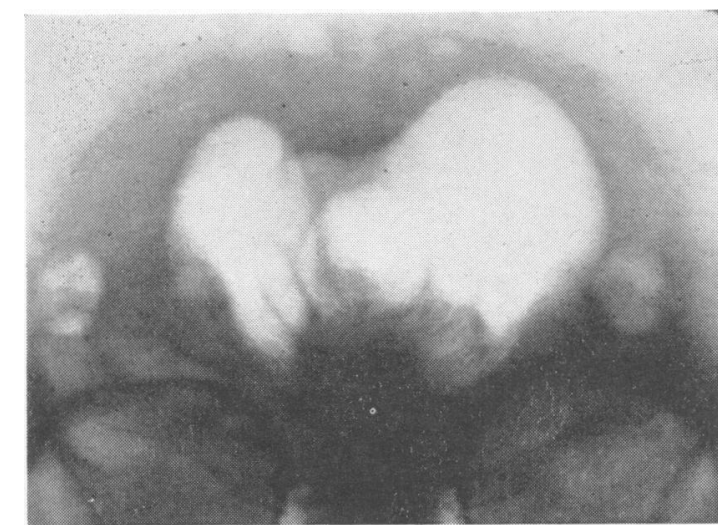

FIG. 1. Defect in the lower part of the right lateral ventricle.

ventricle was reached through a small window in the region of the second frontal gyrus in the plane of the interventricular foramen, and a tumour with an irregular surface was found lying in the lumen of the ventricle. Tampons could be introduced without difficulty between the surface of the tumour and the walls of the ventricle

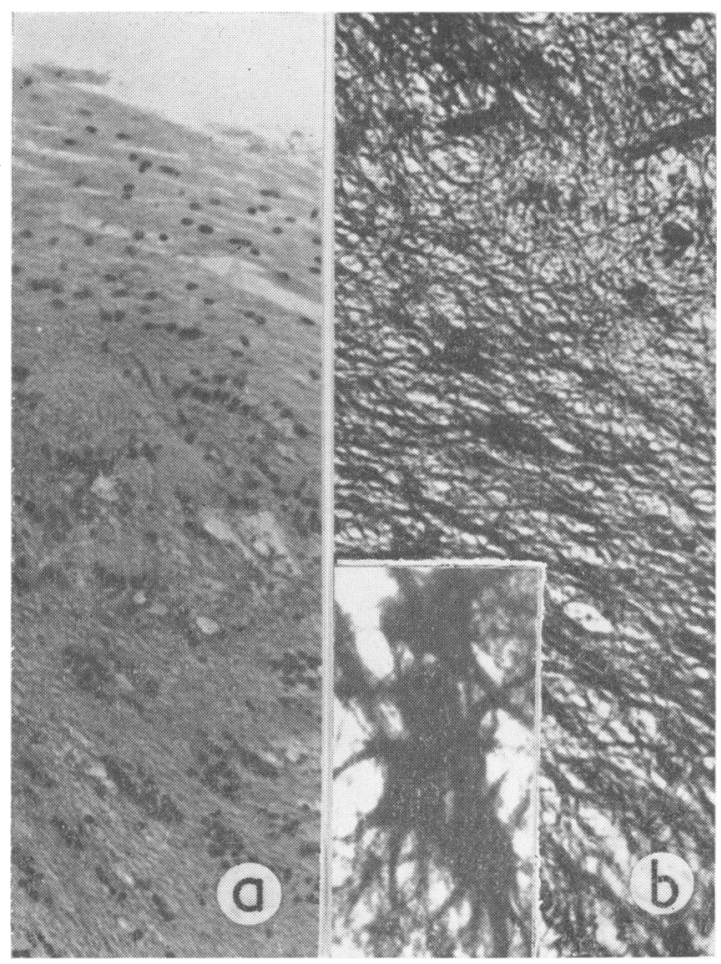

FIG. 2a. Accumulation of minute nuclei preceded by paths of fibrillary glia. Haematoxylin and eosin.

b. Impregnated by Cajal's method. Fibrillary glia. Astrocytes in corner, under high magnification. and it was only in the vicinity of the interventricular foramen that there was found a pedicle about as thick as a match stick, joining the tumour as though by an umbilical cord to the ventricular wall. After severing the pedicle, the tumour $(13.5 \mathrm{ml}$. in volume) was extirpated completely. It was of a hard, dense, cartilaginous consistence. The dura mater was closely sutured and the cranium closed in the usual manner. The post-operative course was smooth.

Seven months after operation the patient returned to his former work as a locksmith in a foundry, where at the time of writing he has been working for nearly four years.

Histopathological examination (no. 3628) showed a picture typical of the tumour described by Scheinker as a subependymoma. In preparations stained with haematoxylin and eosin (Fig. 2), small nuclei are seen placed in elongated columns cut through by tracts of anuclear fibrillary glia. The superficial part of the tumour is composed mainly of glial fibrils among which nuclei are thinly scattered.

In preparations impregnated by Cajal's method it proved that the small groups of cells were composed of minute astrocytes and the non-cellular tracts filled with fibrillary glia. The tumour was scantily vascularized.

CASE 2 B.S.M., aged 50, a fireman (case no. 634/63), was admitted to the clinic on 22 October 1963. For a year he had suffered from pains on the crown of the head lasting some seconds, and for four months had noticed shadows before the eyes and episodic dizziness. During the last two months before admission he had noticed some weakening of the left limbs. Neurological examination showed a very slight weakening of the left limbs and bilateral papilloedema with haemorrhages. The disability of the limbs was so slight that it did not diminish motor efficiency. There were no disturbances in sensation or balance. The patient was completely orientated as to time, place, and situation.

Since the patient had had syphilis, tests were made in this direction. The Wassermann reaction was negative both for the blood and cerebrospinal fluid, but the Lange reaction was markedly pathological (5 6433240 ). Antisyphilitic treatment was given but had no effect whatsoever on the patient's condition. Arteriography furnished no elucidation, and only intraventricular air encephalography showed that there was a tumour causing a filling defect in the base of the anterior part of the right lateral ventricle, which by closing the interventricular foramen had provoked a marked enlargement of the lateral ventricles (Fig. 3). A lateral view shows the posterior extent of the filling defect (Fig. 4).

It was difficult to find a definite explanation for the abnormal pneumoencephalogram, since the possibilities of an intraventricular tumour or a periventricular glioma protruding into the ventricular lumen had to be taken into consideration. Keeping these possibilities in mind, the cranium was opened by a flap revealing the right frontal region, and by incising the second frontal gyrus in the plane of the interventricular foramen the lateral ventricle was reached. After removing the cerebrospinal fluid from the ventricle, a peculiar tumour with an uneven cauli- 


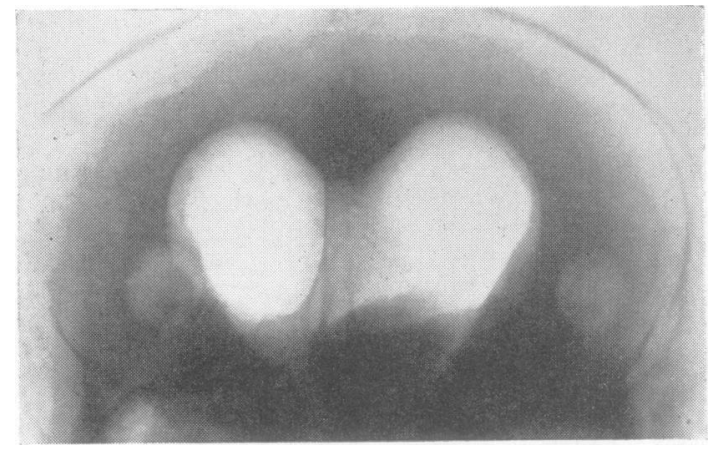

FIG. 3.

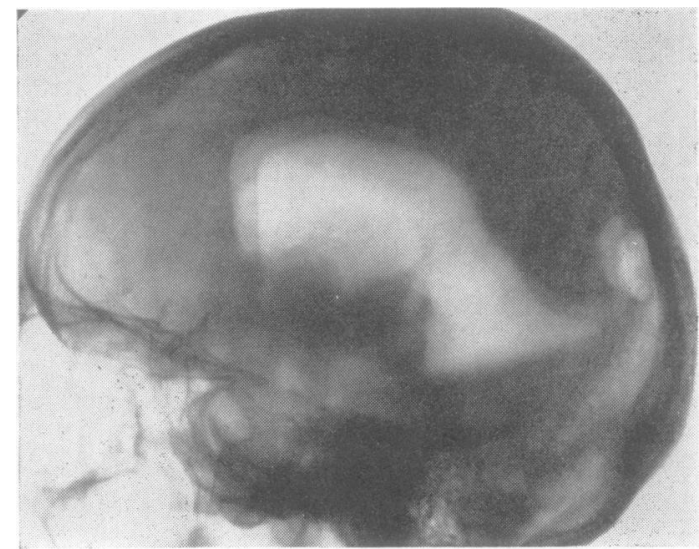

FIG. 4.

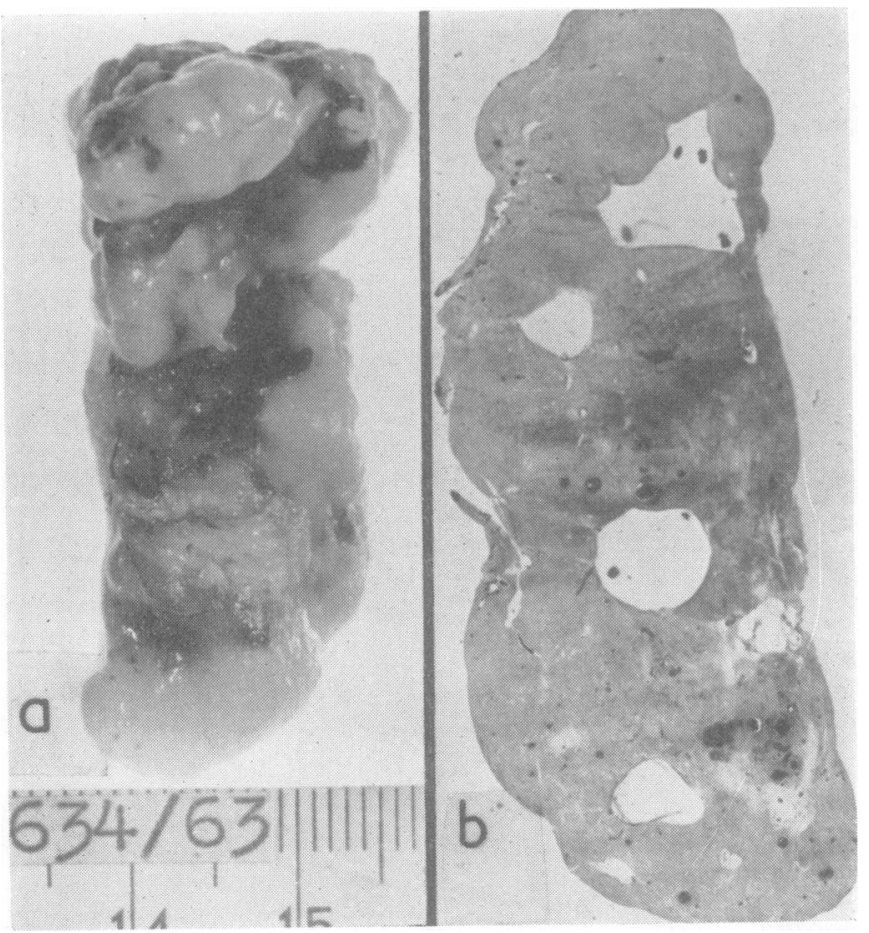

FIG. 3. Defect in the lower parts of the lateral ventricle.

FIG. 4. Wreath-like defect in the lower part of the lumen of the lateral ventricle.

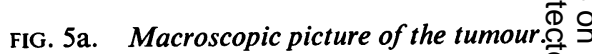

b. Section showing cystic degeneration

FIG. 5 .

flower-like, greyish-white surface was found in the lateral ventricle. Over the pale surface of the tumour there ran numerous tortuous tangled veins. The tumour, of cartilaginous consistency, filled the anterior and medial part of the lateral ventricle, screened the interventricular foramen, and involved the choroid plexus. At operation, it seemed that the surface of the tumour was not joined to the ventricular walls, and the first impression was that the tumour was lying loose in the ventricle.
Closer examination showed the presence of a pedicle 1-2 mm. thick uniting the tumour with the paramedial wall of the ventricle. After placing silver clips in position, the pedicle was severed, and the tumour was lifted out of the ventricle quite freely (Fig. 5). There was no serious haemorrhage. During the application of the clips and the severing of the peduncle, breathing stopped twice for a period of some seconds. The dura mater was firmly sutured and the cranium closed in the usual manner. 


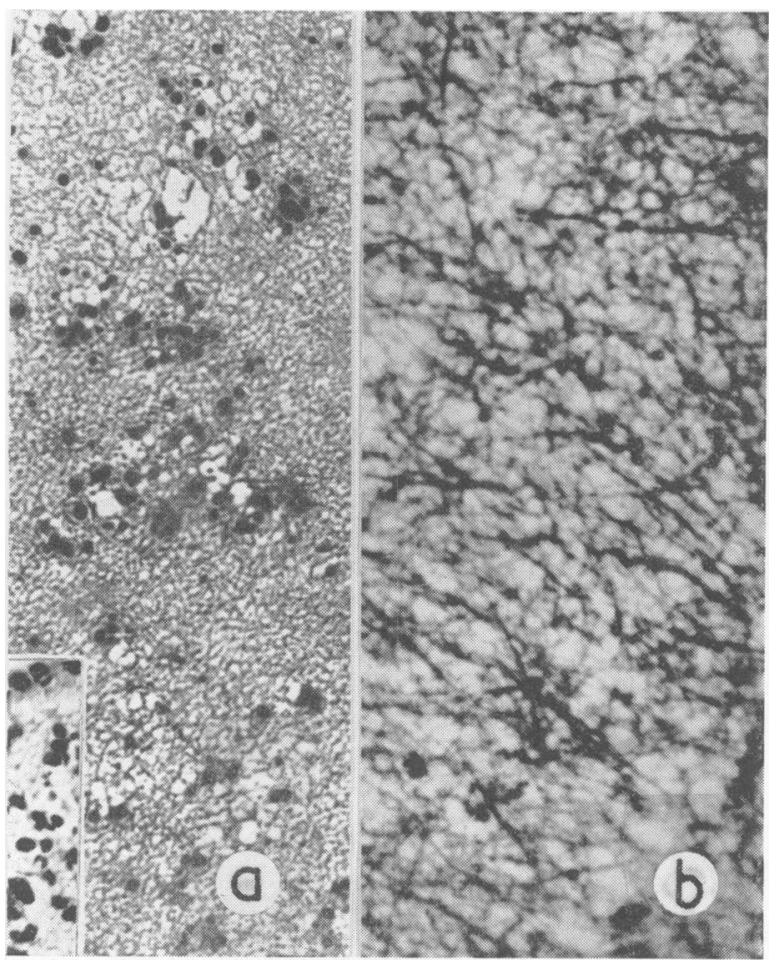

FIG. 6a. Glomerules of small cells in the oedematous tissue. In the corner, the ependymal covering of the surface of the tumour. Haematoxylin and eosin.

b. Bundles of glial fibrils separated by oedema. Cajal's impregnation.

The post-operative course was smooth. After four weeks the patient was discharged from the Clinic, able to walk; now, 13 months after operation, he is working on a farm.

Histopathological examination (no. 4975) showed a microscopic picture of a tumour similar to the previous one. The whole tumour was oedematous, of a spongy appearance, and had undergone degeneration with the formation of small cysts. The groups of minute nuclei were separated by anuclear fields in which fibrillary glia predominated (Fig. 6). Oedematous vesicles clustered round the nuclei. The tumour was very scantily vascularized. In preparations impregnated by Cajal's method, the little groups of cells were seen to be composed of small astrocytes, and the non-cellular tracts were filled with fibrillary glia and were dispersed through the tumour.

The surface of the tumour was in places covered with ependyma. There was a larger accumulation of nuclei in the subependymal layer than in other parts of the growth. The predominance of glial fibrils in the texture explains the hard consistency of the tumour, and the scanty vascularization the tendency to cystic degeneration.

\section{DISCUSSION}

Omitting any evaluation of the suitability of the name given to these tumours by Scheinker, it is difficult not to conclude that they constitute a morphological entity distinct enough to ensure for them a separate place in the systems of classification. Their distinctive morphological character enables these tumours to be differentiated without difficulty from growths diagnosed as ependymal spongioblastoma or ependymoblastoma. Moreover, the special tendency to develop within the ventricular lumen is a peculiarity which is of impcrtance from the point of view of surgical treatment.

Both the cases described form an extreme example of the tendency to grow within a ventricle. In both cases, as in intraventricular tumours of non-glial origin, neurological examination did not indicate the site of the tumour, and for this reason pneumoencephalography was required. It indicated only that the growth occupied part of the ventricular lumen, but gave no answer to the question whether the tumour was developing intraventricularly or whether it originated in the cerebral parenchyma and was only protruding subependymally into the ventricular lumen. Assuming the second possibility, any attempt to remove the growth would have been given up and only palliative measures undertaken, such as Torkildsen's operation. Our cases, in which we may count on a permanent cure, show how mistaken such a procedure would have been. An apparently normal ependyma on the surface of one of the tumours gave the whole growth the appearance of a hamartoma.

\section{SUMMARY}

Two cases of glioma developing in the lumen of a lateral ventricle and joined to the ventricular wall by a peduncle as thick as a match stick are described. In both cases, the site was established by means of penumoencephalography, and all symptoms regressed on removing the tumour. Both patients are working. One patient has remained under postoperative observation for four years and the other for 18 months. The histopathological picture in both cases corresponds to that described by Scheinker as subependymoma.

\section{REFERENCES}

Boykin, F. C., Cowen, D., Iannucci, C. A. J., and Wolf, A. (1954) Subependymal glomerate astrocytomas. J. Neuropath. exp. Neurol., 13, 30-49.

Chason, J. L. (1956). Subependymal mixed gliomas. Ibid., 15, 461-470.

French, J., and Bucy, P. C. (1948). Tumors of the septum pellucidum. $J$. Neurosurg., 5, 433-449.

Russell, D. S., and Rubinstein, L. J. (1959). Pathology of Tumours of the Nervous System. pp. 134-137. Arnold, London.

Scheinker, I. M. (1948). Neurosurgical Pathology, pp. 185-193. Thomas, Springfield, Illinois.

Zülch, K. J. (1956). In Handbuch der Neurochirurgie, edited by H. Olivecrona and W. Tönnis, Vol. 3, p. 334. Springer, Berlin. 